\section{A NEW RULE FOR DIVISION IN ARITHMETIC.}

The ordinary process of long division is rather difficult, owing to the necessity of guessing at the successive figures which form the divisor. In case the repeating decimal expressing the exact quotient is required, the following method will be found convenient.

Rule for division.

First, Treat the divisor as follows :-

If its last figure is a 0 , strike this off, and treat what is left as the divisor.

If its last figure is a 5, multiply the whole by 2 , and treat the product as the divisor.

If its last figure is an even number, multiply the whole by 5 , and treat the product as a divisor.

Repeat this treatment until these precepts cease to be applicable. Call the result the prepared divisor.

Second, From the prepared divisor cut off the last figure; and, if this be a 9, change it to a 1 , or, if it be a 1 , change it to a 9 : otherwise keep it unchanged. Call this figure the extraneous multiplier.

Multiply the extraneous multiplier into the divisor thus truncated, and increase the product by 1 , unless the extraneous multiplier be 7 , when increase the product by 5 . Call the result the current multiplier.

Third, Multiply together the extraneous multiplier and all the multipliers used in the process of obtaining the prepared divisor. Use the product to multiply the dividend, calling the result the prepared dividend.

Fourth, From the prepared dividend cut off the last figure, multiply this by the current multiplier, and add the product to the truncated dividend. Call the sum the modified dividend, and treat this in the same way. Continue this process until a modified dividend is reached which equals the original prepared dividend or some previous modified dividend; so that, were the process continued, the same figures would recur.

Fifth, Consider the series of last figures which have been successively cut off from the prepared dividend and from the modified dividends as constituting a number, the figure first cut off being in the units' place, the next in the tens' place, and so on. Call this the first infinite number, because its left-hand portion consists of a series of figures repeating itself indefinitely toward the left. Imagine another infinite number, identical with the first in the repeating part of the latter, but differing from this in that the same series is repeated unin- terruptedly and indefinitely toward the right, into the decimal places.

Subtract the first infinite number from the second, and shift the decimal point as many places to the left as there were zeros dropped in the process of obtaining the prepared divisor.

The result is the quotient sought.

\section{Examples.}

1. The following is taken at random. Divide 1883 by 365 .

First, The divisor, since it ends in 5, must. be multiplied by 2 , giving 730 . Dropping the 0 , we have 73 for the prepared divisor.

Second, The last figure of the prepared divisor being 3 , this is the extraneous multiplier. Multiplying the truncated divisor, 7, by the extraneous multiplier, 3 , and adding 1 , we have 22 for the current multiplier.

Third, The dividend, 1883, has now to be multiplied by the product of 3 , the extraneous multiplier, and 2 , the multiplier used in preparing the divisor. The product, 11298, is the prepared dividend.

Fourth, From the prepared dividend, 11298, we cut off the last figure, 8 , and multiply this by the current multiplier, 22 . The product, 176 , is added to the truncated dividend, 1129 , and gives 1305 for the first modified divisor. The whole operation is shown thus :-

$$
\begin{aligned}
& 1883 \\
& \frac{6}{11296} \\
& 176^{-} \\
& 1305 \\
& 110 \\
& 2 \longdiv { 4 0 }
\end{aligned}
$$

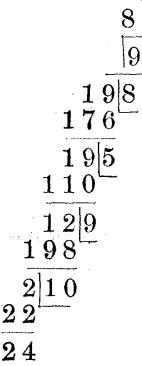

We stop at this point because 24 was a previous modified dividend, written under the form 240 above. Our two infinite numbers (which need not in practice be written down) are, with their difference, -

$$
\begin{aligned}
& \text { i0 }, 958,904,058 \\
& \text { i0,958,904,109.58904i } 0958904 \\
& 51.58904 \text { i } 0958904
\end{aligned}
$$

Hence the quotient sought is $5.1 \dot{5} 890410 \dot{9}$. 
Example 2. Find the reciprocal of 333667. The whole work is here given :-

$$
\begin{array}{r}
33366 \mid 7 \\
233567
\end{array}
$$

Answer, .000002997.

$$
\begin{aligned}
& 16349 \frac{\mid 7}{6 ! 9} \\
& 2102103 \\
& 2265599 \\
& 2102103 \\
& 232866 \mid 2 \\
& 467134 \\
& 700000
\end{aligned}
$$

Example 3. Find the reciprocal of 41 .

$\begin{array}{cc}\text { Solution. }-\frac{4 \mid 1}{37 \mid 9} & \frac{\mid 9}{33 \mid 3} \\ \frac{111}{14 \mid 4} \\ \frac{148}{\left.16\right|^{2}} \\ \frac{74}{90}\end{array}$

Answer, .02439.

\section{S. Peirce.}

\section{URNATELLA GRACILIS, A FRESH- WATER POLYZOAN.}

A PAPER on this polyzoan, by Professor Joseph Leidy, has been recently published, with illustrations, in the Journal of the Academy of natural sciences of Philadelphia. Urnatella was originally discovered in 1851 , and briefly noticed in the Proceedings of the academy the same year, and also subsequently in 1854,1858 , and 1870. It was found in the Schuylkill River at Philadelphia, but has not been seen elsewhere, except a dried but characteristic specimen on the shell of a Unio from Scioto River, Ohio.

Urnatella is an interesting and beautiful form, living in association with Plumatella and Paludicella, and having similar habits, but is very different from them or any other known fresh-water polyzoan, and is most nearly related with the marine genus Pedicellina. It is found attached to the under side of stones beneath which the water can flow. As commonly observed, it consists of a pair of stems divergent in straight lines, or rather gentle curves, from a common disk of attachment. The stems slightly taper, and are beaded in appearance, due to division into segments alternately expanded and contracted. The segments commonly range from two to a dozen, proportioned to the length of the stem, which, when longest, is about the eighth of an inch or a little more. The stems terminate in a bell-shaped polyp, with an expanded oval or nearly circular mouth slanting to one side, and furnished with about sixteen ciliated tentacles. The stems also usually give off a pair of lateral branches from the second segment succeeding the polyp, and frequently likewise from the first segment. The branches consist of a single segment or pedicle supporting a polyp, and usually also give off similar secondary branches. The first and second segments are cylindroid, highly flexible, and mostly striated and colorless, and appear mainly muscular in structure. The succeeding segments are urn-shaped; the body of the urn being commonly pale brown, ringed with lines, and marked with dots of darker brown. The neck and pedicle of the urns are black. The different colors give the stem a beaded and alternately brown and black appearance. Through the lighter colored body of the urns a central cord can be seen, extending through the length of the stem. The urn-shaped segments exhibit lateral pairs of cuplike processes, which correspond in position with the branches from the terminal pair of segments of the stem, and apparently indicate branches which have separated from the parent stem to establish themselves elsewhere as new polyp-stocks.

A series of specimens of Urnatella - from such as consist only of a simple cylindrical, flexible pedicle, supporting a polyp, to those with long stems, consisting of a dozen segments - indicates the urn-shaped segments to be formed successively through segmentation of the originally single simple pedicle. The segments, therefore, do not correspond with what were polyps; but the terminal polyp is permanent, and the segments originate by division from its neck, very much as the segments of the tape-worm arise from its head. After the destruction of the head, the seg-

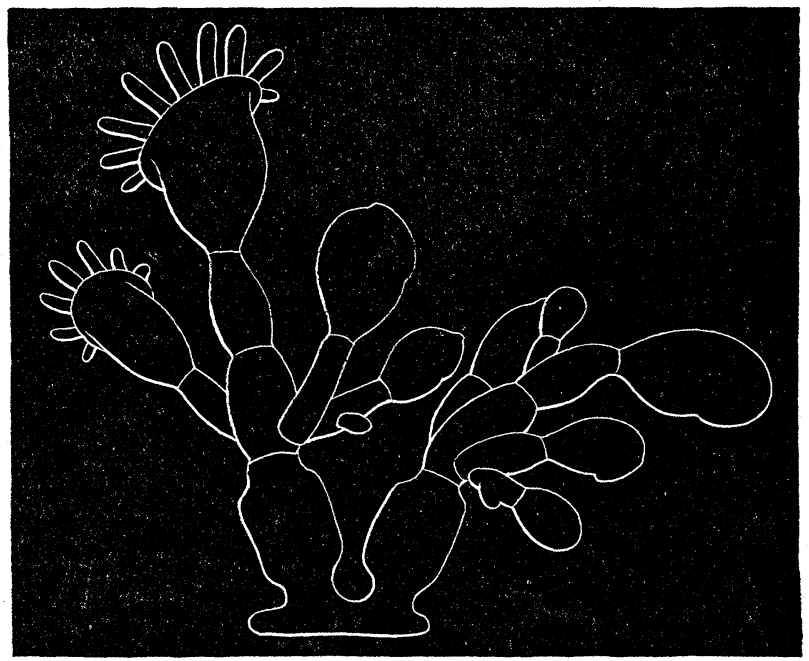

FIG. 1. - Urnatella gracilis. The one on the left with the polyps expanded; that on the right in the condition assumed when the animal is disturbed.

mented stem remains persistent; but what becomes of it ultimately has not been determined. Probably the segments may serve the purpose of the statoblasts of other fresh-water polyzoa, but the question has not 